\title{
Abnormal Methylation of SF-1 Gene Promoter Region in Endometrial Cancer and Its Mechanism
}

\section{Zhen Huang}

The First Affiliated Hospital of Chongqing Medical University

\section{Mingzhu Jia}

The First Affiliated Hospital of Chongqing Medical University

\section{Peng Jiang}

The First Affiliated Hospital of Chongqing Medical University

\section{Ying Deng}

The First Affiliated Hospital of Chongqing Medical University

\section{Shanshan Ding}

The First Affiliated Hospital of Chongqing Medical University

\section{Li Lai}

The First Affiliated Hospital of Chongqing Medical University

\section{Yicheng $\mathrm{Hu}$}

The First Affiliated Hospital of Chongqing Medical University

Zhuoying Hu ( $\sim$ huzhuoying@sina.com )

The First Affiliated Hospital of Chongqing Medical University

\section{Research Article}

Keywords: DNA methylation, Endometrial cancer, Epigenetic regulation, Steroidogenic factor-1, Neoplasms

Posted Date: December 2nd, 2021

DOI: https://doi.org/10.21203/rs.3.rs-1119450/v1

License: (c) (1) This work is licensed under a Creative Commons Attribution 4.0 International License. Read Full License 


\section{Abstract}

We aim to investigate the methylation status, protein expression and clinical significance of the steroidogenic factor-1(SF-1) in endometrial carcinoma (EC), and explore the effect of abnormal methylation of SF-1 on the biological behaviour of EC. Bisulfite sequencing (BSP), western blotting (WB), and immunohistochemical were used to detect the methylation status and protein expression of SF-1 in EC tissues, paracancerous tissues and normal endometrial tissues. DNA methyltransferase inhibitor 5Aza-CdR were used to treat HEC-1-A cell lines to demethylate SF-1. After treatment, WB and QPCR were used to detect the expression of SF-1 and its downstream target genes. Cell proliferation and apoptosis were detected by the EdU fluorescent labelling method and flow cytometry between the groups. Compared with paracancerous tissues and normal endometrial tissues, the expression of SF-1 protein in EC tissues was significantly increased (P凶0.05). The percentage of methylated cytosine in the promoter region of the SF-1 gene in EC tissues (8.2\%) was significantly lower than that in paracancerous tissues by $40.9 \%$ $(P<0.05)$. Compared with the control group, after 5-Aza-CdR treatment, the methylation level of the SF-1 gene was significantly reduced (Pख0.05), the expressions of SF-1 and its downstream target genes were significantly increased $(P<0.05)$, the cell proliferation was enhanced and the cell apoptosis was significantly reduced $(\mathrm{P}<0.05)$. In conclusion, in EC, SF-1 gene was hypomethylated and the expression of SF-1 was increased, which promotes cell proliferation and inhibits cell apoptosis. SF-1 may become a new molecular target for early diagnosis and treatment in EC.

\section{Introduction}

Endometrial carcinoma (EC) is one of the three major malignant tumours of the female reproductive system, and its incidence and mortality are increasing annually, and it is showing a younger trend[1, 2]. The occurrence and development of endometrial cancer is the result of the interaction of various factors. At present, it is believed that long-term estrogen stimulation is the main risk factor leading to endometrial cancer[3].

The steroidogenic factor-1 (SF-1) gene is an orphan nuclear receptor encoded by the Ftz-FI gene, which is one kind of the atypical nuclear receptor [4]. As the main regulator of cholesterol metabolism, SF-1 can activate the cholesterol migration and the expression of genes related to adrenal steroidogenesis and plays an important role in the biosynthesis and development of steroid hormones[5, 6]. Besides, it has been shown in previous studies that abnormal SF-1 expression can lead to abnormal steroidogenesis, which promotes the malignant progression of glandular tumours and hormone-related diseases, such as adrenal tumours, ovarian tumours and endometriosis, etc. Current studies have suggested that the increased expression of SF-1 should be used as a key indicator to predict adrenal tumours [7-9].

According to previous studies, there are epigenetic regulation abnormalities of certain genes in endometrial cancer, which plays an important role in the occurrence and development of EC[10]. Among them, DNA methylation, especially abnormal methylation of CpG islands in the promoter region of genes has become a research hotspot[11, 12]. Tumor-related gene hypomethylation is an early epigenetic 
change in the process of tumorigenesis, which promotes tumorigenesis by activating the expression of some oncogenes or related factors that are usually suppressed and affecting the stability of chromosomes[13]. Studies have shown that the expression of SF-1 is also regulated by DNA methylation, but there are few studies on the role of SF-1 in endometrial cancer $[14,15]$.

This study detected the methylation status of SF-1 promoter and the expression of SF-1 gene in EC tissues, and analyzed its relationship with EC clinicopathological parameters, and explored the clinical significance of the methylation status of SF-1 promoter and gene expression in the occurrence and development of EC. And we further investigated the effects of abnormal methylation of SF-1 gene promoter on the expression of SF-1 expression and biological behaviour of endometrial cancer cells and its possible molecular mechanism. It is expected to provide an experimental and theoretical basis for finding an effective diagnostic biomarker and treatment strategies in endometrial cancer.

\section{Methods}

\subsection{Materials}

60 cases of tissue specimens were diagnosed as EC according to the International Federation of Gynecology and Obstetrics (FIGO) standards in the department of gynaecology at the first affiliated hospital of Chongqing medical university from January 2013 to August 2014. Among them, 30 cases of paracancerous tissues were collected at the same time. In addition, 20 cases of normal endometrial tissues with diagnostic curettage due to dysfunctional uterine bleeding were collected. The age of patients, surgical stage, degree of differentiation, pathological types, lymph node metastasis were recorded. None of the patients received radiotherapy or chemotherapy before surgery. All sample collections were approved by the ethics committee of Chongqing Medical University (2016-133) and informed consent with patients and their family members.

\subsection{Cell culture}

The endometrial cancer cell line HEC-1-A was purchased from Shanghai Institute of Cell Biology, Chinese Academy of Sciences (Shanghai, China). Take out the endometrial cancer cell HEC-1-A cryopreservation tube from the liquid nitrogen tank and place it in a preheated water bath to thaw the cells for cell resuscitation and passage. The cells in the logarithmic growth phase were seeded on the cell culture plate. 24 hours after seeding, different concentrations of 5-Aza-CdR working solution (1.0uM, 2.5uM, 5.0uM, 10uM, 15uM) were added, and each cell line was divided into Different concentrations of 5-Aza$\mathrm{CdR}$ group and control group without any treatment. Open for $96 \mathrm{~h}$ in a sterile incubator with a constant temperature of $37^{\circ} \mathrm{C}, 5 \% \mathrm{CO} 2$ and saturated humidity (change the fresh working fluid every day), and collect the cells for subsequent experiments.

\subsection{Immunohistochemistry (IHC).}


Fix the tissue sample in $40 \%$ formalin, then embed it in a paraffin block, and cut $3 \mu \mathrm{m}$ slices. The tissue sections were sequentially placed in xylene and gradient alcohol for deparaffinization and hydration. Incubate with $3 \%$ hydrogen peroxide ( $\mathrm{H} 2 \mathrm{O} 2)$ for 30 minutes at room temperature to block endogenous peroxidase, and add an appropriate amount of citrate buffer for antigen retrieval. After washing with PBS, add goat serum for 30 minutes at room temperature to reduce non-specific binding. Add rabbit antihuman SF-1 polyclonal antibody (primary antibody) $₫ A b c a m$ Inc., UK囚, overnight at $4^{\circ} \mathrm{C}$, wash and then add horseradish-labelled goat anti-rabbit secondary antibody $₫$ Biyuntian Biotechnology Co., Ltd., Shanghai, China冈to incubate, and finally use 3,3'-diaminobenzidine (DAB) colour reagent kit (Golden Bridge International Inc., Beijing, China) for visualization. Hematoxylin was used for staining, and the sealed specimens were placed under a microscope for inspection. Refer to Shimizu's method for the result judgment: the number of positive cells: no positive staining is 0 , the number of positive cells $<1 / 3$ is 1 , the number of positive cells $>2 / 3$ is 3 , and the number of positive cells is 2 in $1 / 3 \otimes 2 / 3$. Judgment criteria for staining intensity: cells are not stained as 0 , strong staining is 2 , and moderate staining (between 0 and 2$)$ is 1 . The above two points are added to the final result: 0 points means $(-), 2$ points means $(+), 3$ points means $(++)$, and 4 points means $(+++)$. Among them, $(-)$ is negative expression, $(+)(++)(+++)$ is positive expression.

\subsection{Western blot analysis}

The lysate containing protease inhibitors $₫$ Biyuntian Biotechnology Co., Ltd., Shanghai, China囚is added to extract tissue cell proteins. Use the BCA kit\Biyuntian Biotechnology Co., Ltd., Shanghai, China冈to determine the protein concentration. Completely mix $32 \mu \mathrm{l}$ of sample protein and $8 \mu$ l of $5 \times$ SDS-PAGE buffer for electrophoresis, transfer the membrane to PVDF membrane (Millipore Corp, MA, USA), seal with $5 \%$ skimmed milk powder and incubate at room temperature for 1 hour. Then incubate with rabbit antihuman SF-1 polyclonal antibody $₫ A b c a m$ Inc., UKखat $4^{\circ} \mathrm{C}$ overnight. After washing the next day, place it in a blocking buffer containing horseradish-labelled goat anti-rabbit secondary antibody $₫$ Biyuntian Biotechnology Co., Ltd., Shanghai, China囚and incubate for 2 hours. Use ECL kit (Sigma Inc., MO, USA) for enzyme-linked chemiluminescence. Analyze by Quantity One software, and use the ratio of the target band to the internal reference band to express the result.

\subsection{Bisulfite-sequencing PCR}

Genomic DNA extraction and purification was performed using the Genomic DNA Purification kit (Tiangen Biochemical Technology Co., Ltd., Beijing, China) according to the manufacturer protocol. Then the extracted DNA was subjected to sodium bisulfite modification: A-2ug DNA sample was denatured in 5.5ul of $3 \mathrm{M} \mathrm{NaOH}$, then followed by $30 \mathrm{ul}$ of $10 \mathrm{mM}$ hydroquinone, $520 \mathrm{ul}$ of $3.6 \mathrm{M}$ sodium bisulfite, and $200 \mathrm{ul}$ of paraffin oil. The mixture was then incubated in a water bath at $50{ }^{\circ} \mathrm{C}$ for 16 hours. The DNA sample was desulphonated in $3 \mathrm{M} \mathrm{NaOH}$ and ethanol precipitated. Primers were designed using the bisulfitetreated DNA as a template, and a PCR amplification machine (Eppendorf Company, Hamburg, Germany) was used for the PCR reaction of the target gene. The primer sequences are were as follow: M12-F 5'GTGYGGGGATAAGGTGTTYGGTT 3'; M12-R 5'CTTCCAAACRCATCCCCACC 3', all primers were 


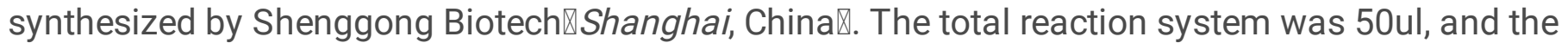
reaction conditions were as follows: pre-denaturation at $98^{\circ} \mathrm{C}$ for $4 \mathrm{~min}$, two cycles of denaturation at $94^{\circ} \mathrm{C}$ for $45 \mathrm{sec}$, annealing at $66^{\circ} \mathrm{C}$ for $45 \mathrm{sec}$, extension at $72^{\circ} \mathrm{C}$ for 1 minute, followed by extension at $72^{\circ} \mathrm{C}$ for 8 minutes at the end of the cycles. Gel electrophoresis on the PCR product was performed according to the PCR product purification and recovery kit (Shenggong Biotech, Shanghai, China) Next, connect it to a plasmid vector by TA cloning method, and then transform it into competent cells. The white colonies grown on the IPTG/X-gal plate were inoculated into liquid medium containing ampicillin. Finally, the methylation status of the target fragment was detected.

\subsection{Cell proliferation assay}

HEC-1-A cells in the logarithmic growth phase were collected and seeded in 96-well plates at 4000 cells/well, following incubation for $24 \mathrm{~h}$, and then treated with different concentrations (1.0uM $2.5 \mathrm{uM} \mathbb{\mathrm { V }}$ 5.0uM $\triangle 10 \mathrm{uM} \otimes 15 \mathrm{uM}) 5-A z a-C d R .50 \mu \mathrm{L}$ diluted EdU solution was added to each well and incubated for $2 \mathrm{~h}$. The cells were fixed and stained based on the instructions of the EdU kits (Guangzhou RiboBio Co., Ltd. Guangdong, China). Subsequently, cell proliferation was observed based on the ratio of the number of red fluorescent cells to the total number of cells in the field of view.

\subsection{Flow cytometry analysis}

After inoculation for $24 \mathrm{~h}$, the HEC-1-A cells were treated with 5-Aza-CdR at concentrations of 1.0uM, $5.0 \mathrm{uM}$ for $96 \mathrm{~h}$. Cells were dissociated with trypsin and resuspended at $1 \times 10^{6} \mathrm{cells} / \mathrm{mL}$ in binding buffer. Then $100 \mu \mathrm{L}$ cell suspension was incubated with $5 \mu \mathrm{L}$ Annexin V/FITC (Beyotime Institute of Biotechnology, Shanghai, China $₫$ and $5 \mu \mathrm{L}$ PI solution at room temperature for 15-20 mins and then were analyzed flow cytometer (Becton Dickinson, USA).

\section{8 qPCR detection of SF-1 downstream gene mRNA expression}

The total RNA was extracted by the Trizol method, and the OD260/OD280 value was determined. After being reversed to $\mathrm{CDNA}$, it was stored at $-20^{\circ} \mathrm{C}$ for later use. PCR amplification adopts real-time fluorescent quantitative PCR instrument (Mx3000p type) (Eppendorf Company, Hamburg, Germany), and the reaction conditions are $94^{\circ} \mathrm{C} 5 \mathrm{~min} ;\left(94^{\circ} \mathrm{C} 30 \mathrm{~s} ; \mathrm{Tm} 30 \mathrm{~s} ; 72^{\circ} \mathrm{C} 30 \mathrm{~s}\right) 40$ cycles; $72^{\circ} \mathrm{C} 10 \mathrm{~min}$. The qPCR data were statistically analyzed by the $2-\triangle \triangle \mathrm{CT}$ method. The primer sequence is shown in Table 1 .

\subsection{Statistical analysis}

The statistical analysis of data uses SPSS 26.0 software, and the measurement data is expressed as $\mathrm{x} \pm \mathrm{s}$. The normality test and the homogeneity of variance test were performed on each group of data. The enumeration data adopts the $\chi 2$ test. T-test was used to analyze the difference between two groups, and the difference between multiple groups was analyzed by analysis of variance. $P<0.05$ was considered statistical significance. 


\section{Results}

\subsection{Expression of SF-1 protein in EC tissues}

The expression of SF-1 protein in EC tissue [100\% (60/60)] is significantly higher than that of adjacent tissues $[30 \%(9 / 30)]$ and normal endometrial tissue [15\% (3/20)], the differences are statistically significant $(X 2=8.22, P=0.038 ; X 2=9.21, P=0.015)$, but there is no statistically significant difference between paracancerous tissues and normal endometrial tissue $(X 2=4.11, P=0.251)$. The results of $I H C$ and Western blot are shown in Figure 1 and Figure 2.

\subsection{Expression of SF-1 protein in different clinicopathological characteristics of EC tissue}

The results show that the worse the differentiation of EC tissue, the higher the expression level of SF-1 protein, and the difference in the expression between different tissues is statistically significant (Pख0.05). Compared with endometrioid adenocarcinoma, the expression of SF-1 in special types of EC was significantly higher (P凶0.05). However, the expression of SF-1 protein was not significantly different in different ages, surgical pathological stages, depth of myometrial invasion and lymph node metastasis $(P>0.05)$. See Table 2.

\subsection{The expression of DNA methyltransferase (DNMT) in EC tissues}

Western blot results showed that there was no statistically significant difference in the expression of DNMT1 protein in EC and adjacent tissues $(P>0.05)$. The expression of DNMT3a and DNMT3b in EC tissues was significantly lower than that of adjacent tissues, and the difference was statistically significant $(P<0.05)$. See Figure 3.

\subsection{The methylation status of the promoter region of the SF-1 gene in EC tissues}

The percentage of methylated cytosine in EC tissue [8.2\% (9/110)] was significantly lower than that in adjacent tissues [40.9\% (27/66)], and the difference was statistically significant (X2=8.94, $P=0.029)$. Figure 4 shows the results of BSP detection of the methylation status of cytosine (C) at each CpG site in the promoter region of the SF-1 gene in EC and adjacent tissues.

\subsection{Cell proliferation in endometrial cancer cells after 5-Aza-CdR treatment}

The endometrial cancer cell line HEC-1-A was treated with different concentrations of 5-Aza-CdR, and the ratio of EdU positive cells was compared with the control group. It showed that 1.0uM 5-Aza-CdR can induce cell proliferation compared with the control group $(P<0.05)$, while other concentration groups inhibited the proliferation due to $5-A z a-C d R$ cytotoxicity $(P<0.05)$. The results were shown in Figure 5 .

\subsection{The expression of SF-1 protein after 5-Aza-CdR treatment}

The result of Western blot analysis was shown in Figure 6. It indicated that SF-1 protein expressed at a low level in the HEC-1-A cell line without treatment. After being treated with 4 different concentrations 
$(1 \mathrm{uM}, 2.5 \mathrm{uM}$, and 5.0uM) of 5-Aza-CdR, the expression of SF-1 protein in HEC-1-A cells was significantly increased in all groups compared with the control group $(P<0.05)$.

\subsection{Methylation status of SF-1 gene promoter after 5-Aza-CdR treatment}

The BSP method was used to detect the methylation status of cytosine at $22 \mathrm{CpG}$ sites in the SF-1 gene promoter region of endometrial cancer cells HEC-1-A cells. The results showed that the background level of SF-1 gene promoter in HEC-1-A cells was highly methylated, and the percentage of methylated cytosine was $87.3 \%$. Compared with the background level, $1.0 u M$ (other concentrations) 5-Aza-CdR treated cells for $96 \mathrm{~h}$ significantly reduced the methylation level of the SF-1 gene promoter region of HEC-1-A cells (56.4\%) $(P<0.05)$ (Figure 7).

\subsection{5-Aza-CdR regulates the mRNA expression of SF-1 downstream genes}

The qPCR method was used to detect the mRNA expression of SF-1 downstream target genes StAR, HSD3 32 and CYP19A1 after treating endometrial cancer cells with various concentrations of 5-Aza-CdR. Compared with the control group, except for HEC-1-A cells treated with 1.0uM 5-Aza-CdR group, there was no change in the expression of SF-1 downstream gene StARmRNA. After 5-Aza-CdR treatment in the other groups, StAR, HSD3 32 and CYP19A1 mRNA in endometrial cancer HEC-1-A cells were significantly increased $(P<0.05)$. See Figure8.

\subsection{Cell apoptosis in endometrial cancer cells after 5-Aza-CdR treatment}

After treating HEC-1-A cells with 5-Aza-CdR, the cells were collected by centrifugation for flow cytometric detection of apoptosis. The results are shown in Figure 9. Compared with the control group, the apoptosis rate of the 1.0 uM5-Aza-CdR treatment group was significantly decreased, and the apoptosis rate of the $5.0 \mathrm{uM}$ treatment group was significantly increased $(P<0.05)$. It shows that cell apoptosis is inhibited after treatment with a low concentration of 5-Aza-CdR; while the high concentration of 5-Aza-CdR has a toxic effect on cells and increases cell apoptosis.

\subsection{The expression of apoptosis-related proteins and CyclinD3}

After 1.0uM5-Aza-CdR treatment of HEC-1-A, the expression of apoptosis-related protein Bcl-2 increased significantly $(P<0.05)$, and the ratio of Bcl-2/Bax increased. After 5.0 uM5-Aza-CdR treatment, the expression of Bax was extremely significant Increased $(P<0.01)$, the $B c l-2 / B a x$ ratio decreased, and the expression of Caspase-3 was significantly increased $(P<0.05)$ (Figure 10).

After 1.0uM and 2.5uM 5-Aza-CdR treatment, the expression of cyclin Cyclin D3 in HEC-1-A cells was significantly increased $(P<0.05)$. After the 5-Aza-CdR concentration was increased to $5 \mathrm{uM}$ and $10 \mathrm{uM}$, the expression of Cyclin D3 did not change significantly (Figure 11).

\section{Discussion}


DNA methylation is an epigenetic modification mechanism. The inactivation of tumour suppressor genes and the activation of oncogenes caused by abnormal methylation of its promoter are involved in the occurrence and development of tumours [16]. Studies have found that the promoter methylation abnormalities of many key genes are involved in the occurrence and development of EC[17]. Therefore, it is important to explore the methylation status of some key genes in EC, use these abnormal genes methylation as biomarkers for precancerous stage and early cancer diagnosis, and provide references for evaluating the efficiency and judging prognosis.

SF-1 is a member of the nuclear receptor superfamily that regulates steroid production, which is essential to maintain the normal development and function of the adrenal glands and steroid-producing gonads[18, 19]. Studies believe that the abnormal expression of SF-1 gene promotes the malignant progression of adrenal tumours, and is considered to be an important biomarker for the prognostic evaluation of adrenal tumours $[20,21]$. Studies of endometriosis have found that the level of SF-1 mRNA in endometriotic stromal cells is significantly higher than that in endometrial stromal cells and further found that the $\mathrm{CpG}$ islands in the SF-1 promoter region are highly methylated in endometrial stromal cells, while the lack of methylation in endometriotic stromal cells leads to abnormally high levels of SF-1 expression[22, 23]. Both EC and endometriosis are related to estrogen exposure[24, 25]. Therefore, we speculate that the abnormal methylation status of the SF-1 gene promoter may be related to the pathogenesis of EC.

Our study suggests that compared with paracancerous tissues and normal endometrial tissues, EC tissues have a higher expression of SF-1 protein. Further analysis of the relationship between SF-1 protein expression and tumour clinicopathological characteristics found that the poorer the tissue differentiation and special pathological types, the higher the expression of SF-1 protein in EC tissues. The poorly differentiated tumour tissues and special pathological types are recognized as high-risk factors related to EC recurrence[26], which suggests that the abnormal expression of SF-1 protein may be related to the poor prognosis of EC. The DNA methyltransferase (DNMT) family is a key molecule that regulates DNA methylation. It plays an important role in the initiation and maintenance of DNA methylation in tumour cells. It is related to the methylation and inactivation of tumour suppressor genes in malignant tumours and the development, invasion and metastasis of tumours [27]. Compared with the adjacent tissues, the methylation level of the SF-1 promoter and the methylation-related proteins DNMT3a and DNMT3b in EC tissues are lower in EC tissues. This suggests that SF-1 gene hypomethylation is tumour-specific in EC, which may be the reason for the high expression of SF-1 protein in tumour tissues. The low expression of DNMT3a and DNMT3b may be involved in the hypomethylation mechanism of SF-1 gene. According to reports, mammalian catalysis of new methylation patterns is achieved through DNMT3a and DNMT3b[28]. However, the results of this study showed that there was no difference in the expression of DNMT1 in EC and adjacent tissues. This may be because the main function of DNMT1 is to maintain the organic DNA methylation pattern, while DNMT3a and DNMT3b are related to abnormal methylation and catalyzing new methylation patterns in tumour tissues[29]. Specific genes may have abnormal methylation at different stages of tumour development, which allows us to detect malignant tumours at an early stage of the disease by detecting the methylation status of specific genes or assess the risk of 
disease progression to early tumours [30]. In addition, the methylation status of genes is reversible, which makes it possible for us to affect the expression of specific genes by changing the methylation status of specific genes in the precancerous stage, providing new ideas for tumour treatment. Using gene methylation status as a biomarker also has a predictive effect. Monitoring DNA methylation after treatment may provide evidence for disease recurrence. Therefore, it is worthy of exploration of the abnormal methylation status of key genes in EC.

In order to further clarify the effect of changes in SF-1 methylation status on SF-1 expression, this study used DNA methyltransferase inhibitors (5-Aza-CdR) to treat endometrial cancer cells and found the methylation level of the promoter region of SF-1 gene was significantly reduced, and the expression of SF1 protein was significantly increased in HEC-1-A cells, which indicated that the expression of SF-1 was regulated by methylation in EC cells. When the promoter of SF-1 gene was demethylated the expression of SF-1 is up-regulated. Hoivik also found the methylation status of the SF-1 gene promoter has a direct regulatory effect on the expression of SF-1 in the cell research of mice and humans[14]. This means that in endometrial cancer or precancerous stage, the expression of the SF-1 gene may be affected by changing the methylation status of the SF-1 gene, to achieve the purpose of treating and preventing tumours. It is worth mentioning that, the expression level of SF-1 protein did not increase stepwise after treatment with different concentrations of 5-Aza-CdR, and even showed a downward trend when the inhibitor reached a certain concentration. This may be due to the inhibitory receptors in cells have reached saturation after a certain concentration, and high concentrations of 5-Aza-CdR also have drug toxicity on cells, thereby affecting the expression of SF-1 protein in cells. In our experiment, the SF-1 protein expression was significantly higher than that of the control group after treatment with a low concentration of 5-Aza-CdR, which is enough to indicate that the methylation status of the SF-1 gene promoter directly regulates the expression of SF-1 protein.

Studies have reported that the SF-1 gene plays an important regulatory role in the cell cycle, cell proliferation and apoptosis[21]. In vitro studies have reported that human adrenal cortex cells H295R after being artificially transfected with SF-1 plasmid can promote cell differentiation and proliferation while inhibiting cell apoptosis. In mice, SF-1 transgene can induce adrenal hyperplasia and adrenal cortex tumours while after removing the SF-1 gene, the proliferation of H295R cells slowed down[31]. In this study, through comparative analysis before and after drug treatment, we found that after treatment of cells with a low concentration of 5-Aza-CdR demethylated SF-1, cell proliferation increased and apoptosis decreased. At the same time, the expression of apoptosis-related protein and cyclin Cyclin D3 increased significantly after SF-1 gene demethylation, which further shows that the hypomethylation state of SF-1 gene can regulate local steroid hormone synthesis and promote the conversion of the cell growth cycle. As a result, the normal endometrial tissue received excessive estrogen stimulation, which in turn promotes proliferation, inhibits apoptosis, and promotes the occurrence and development of endometrial cancer. This study proves that the SF-1 gene has the characteristics of an oncogene in endometrial cancer, regulates the biological behaviour of tumour cells, and may play an important role in the occurrence and development of endometrial cancer. The methylation level of SF-1 may be a predictor of the proliferation of EC. Therefore, it is expected that the targeted use of SF-1 inverse agonist to reverse the high expression 
of SF-1 by detecting the level of SF-1 methylation in endometrial cancer, to develop individualized diagnosis and treatment strategies to achieve the purpose of improving prognosis.

In summary, this study confirmed that the promoter region of the SF-1 gene in EC tissues was hypomethylated, and the SF-1 protein was significantly overexpressed. It proved that changes in the methylation status of the SF-1 gene can regulate gene expression and cause a series of biological behaviour changes of endometrial cancer cells, which may play an important role in the occurrence and development of EC. SF-1 methylation level may become a new molecular target for early diagnosis and prognosis assessment of endometrial cancer.

\section{Declarations}

Running Title: Z Huang et al: SF-1 hypomethylation participates in endometrial cancer

Funding: This work was supported by the Young and Middle-age High-level Medical Reserved Personnel Training Project Foundation of Chongqing, China [2016HBRC002].

Availability of data and materials: The datasets used and analyzed during the current study are available from the corresponding author on reasonable request.

\section{Authors' contributions}

ZY Hu conceived and designed research; Z Huang and MZ Jia performed experiments; Z Huang and MZ Jia analyzed data; $Z$ Huang, $P$ Jiang and MZ Jia interpreted results of experiments; $Y$ Deng, $L$ Lai and SS Ding prepared figures; $Z$ Huang and $M Z$ Jia drafted manuscript; $P$ Jiang, $Y$ Deng, SS Ding, $L$ Lai and $Y C$ Huedited and revised manuscript; ZY Hu and MZ Jia confirm the authenticity of all the raw data. Z Huang, MZ Jia, P Jiang, Y Deng, SS Ding, L Lai, YC Hu and ZY Hu approved final version of manuscript.

Ethics approval and consent to participate: Our study was approved by the ethics committee of Chongqing Medical University (2016-133) and informed consent with patients and their family members.

Patient consent for publication: All patients gave written informed consent before participation in this study.

Competing interests: The authors declare that they have no competing interests

\section{Acknowledgements}

Thanks to the Young and Middle-age High-level Medical Reserved Personnel Training Project Foundation of Chongqing, China [2016HBRC002].

\section{References}


[1] Miller MD, Salinas EA, Newtson AM, et al. An integrated prediction model of recurrence in endometrial endometrioid cancers. Cancer Manag Res. 2019;11:5301-15.

[2] De Boer SM, Nout RA, Bosse T, Creutzberg CL. Adjuvant therapy for high-risk endometrial cancer: recent evidence and future directions. Expert Rev Anticancer Ther. 2019;19(1):51-60.

[3] Liu Y, Zhao R, Chi S, et al. Is Upregulated by Estrogen and Promotes Epithelial-Mesenchymal Transition via p53 in Endometrial Cancer. Mol Cancer Res. 2020;18(2):204-15.

[4] Emura N, Wang C-M, Yang WH, Yang W-H. Steroidogenic Factor 1 (NR5A1) Activates ATF3 Transcriptional Activity. Int J Mol Sci. 2020;21(4).

[5] Hammer GD, Ingraham HA. Steroidogenic factor-1: its role in endocrine organ development and differentiation. Front Neuroendocrinol. 1999;20(3):199-223.

[6] Wong M, Ikeda Y, Luo X, et al. Steroidogenic factor 1 plays multiple roles in endocrine development and function. Recent Prog Horm Res. 1997;52.

[7] Lichtenauer UD, Duchniewicz M, Kolanczyk M, et al. Pre-B-cell transcription factor 1 and steroidogenic factor 1 synergistically regulate adrenocortical growth and steroidogenesis. Endocrinology. 2007;148(2):693-704.

[8] Sekiya A, Takasawa K, Arai Y, Torisu S, Nishino K. Dog Steroidogenic Factor-1: Molecular cloning and analysis of epigenetic regulation. J Vet Med Sci. 2020;82(6):681-9.

[9] Figueiredo BC, Cavalli LR, Pianovski MAD, et al. Amplification of the steroidogenic factor 1 gene in childhood adrenocortical tumors. J Clin Endocrinol Metab. 2005;90(2):615-9.

[10] Zhong Q, Fan J, Chu H, et al. Integrative analysis of genomic and epigenetic regulation of endometrial cancer. Aging (Albany NY). 2020;12(10):9260-74.

[11] Kommoss FKF, Stichel D, Schrimpf D, et al. DNA methylation-based profiling of uterine neoplasms: a novel tool to improve gynecologic cancer diagnostics. J Cancer Res Clin Oncol. 2020;146(1).

[12] van den Helder R, Wever BMM, van Trommel NE, et al. Non-invasive detection of endometrial cancer by DNA methylation analysis in urine. Clin Epigenetics. 2020;12(1):165.

[13] De Smet C, Loriot A. DNA hypomethylation in cancer: epigenetic scars of a neoplastic journey. Epigenetics. 2010;5(3):206-13.

[14] Hoivik EA, Bjanesoy TE, Mai O, et al. DNA methylation of intronic enhancers directs tissue-specific expression of steroidogenic factor 1/adrenal 4 binding protein (SF-1/Ad4BP). Endocrinology. 2011;152(5):2100-12. 
[15] Matsukura H, Aisaki K-i, Igarashi K, et al. Genistein promotes DNA demethylation of the steroidogenic factor 1 (SF-1) promoter in endometrial stromal cells. Biochem Biophys Res Commun. 2011;412(2):36672.

[16] Skvortsova K, Stirzaker C, Taberlay P. The DNA methylation landscape in cancer. Essays Biochem. 2019;63(6):797-811.

[17] Tao MH, Freudenheim JL. DNA methylation in endometrial cancer. Epigenetics. 2010;5(6):491-8.

[18] Luo X, Ikeda Y, Parker KL. A cell-specific nuclear receptor is essential for adrenal and gonadal development and sexual differentiation. Cell. 1994;77(4):481-90.

[19] Val P, Lefrançois-Martinez A-M, Veyssière G, Martinez A. SF-1 a key player in the development and differentiation of steroidogenic tissues. Nucl Recept. 2003;1(1):8.

[20] Almeida MQ, Soares IC, Ribeiro TC, et al. Steroidogenic factor 1 overexpression and gene amplification are more frequent in adrenocortical tumors from children than from adults. $\mathrm{J}$ Clin Endocrinol Metab. 2010;95(3):1458-62.

[21] Doghman M, Karpova T, Rodrigues GA, et al. Increased steroidogenic factor-1 dosage triggers adrenocortical cell proliferation and cancer. Mol Endocrinol. 2007;21(12):2968-87.

[22] Xue Q, Zhou YF, Zhu SN, Bulun SE. Hypermethylation of the $\mathrm{CpG}$ island spanning from exon II to intron III is associated with steroidogenic factor 1 expression in stromal cells of endometriosis. Reprod Sci. 2011;18(11):1080-4.

[23] Xue Q, Xu Y, Yang H, et al. Methylation of a novel CpG island of intron 1 is associated with steroidogenic factor 1 expression in endometriotic stromal cells. Reprod Sci. 2014;21(3):395-400.

[24] Marquardt RM, Kim TH, Shin J-H, Jeong J-W. Progesterone and Estrogen Signaling in the Endometrium: What Goes Wrong in Endometriosis? Int J Mol Sci. 2019;20(15).

[25] Zhou W-J, Zhang J, Yang H-L, et al. Estrogen inhibits autophagy and promotes growth of endometrial cancer by promoting glutamine metabolism. Cell Commun Signal. 2019;17(1):99.

[26] Jiang P, Huang J, Deng Y, et al. Predicting Recurrence in Endometrial Cancer Based on a Combination of Classical Parameters and Immunohistochemical Markers. Cancer Manag Res. 2020;12:7395-403.

[27] Lyko F. The DNA methyltransferase family: a versatile toolkit for epigenetic regulation. Nat Rev Genet. 2018;19(2):81-92.

[28] Tajima S, Suetake I, Takeshita K, Nakagawa A, Kimura H. Domain Structure of the Dnmt1, Dnmt3a, and Dnmt3b DNA Methyltransferases. Adv Exp Med Biol. 2016;945:63-86. 
[29] Chen Z, Zhang Y. Role of Mammalian DNA Methyltransferases in Development. Annu Rev Biochem. 2020;89:135-58.

[30] Liang G, Weisenberger DJ. DNA methylation aberrancies as a guide for surveillance and treatment of human cancers. Epigenetics. 2017;12(6):416-32.

[31] Lucki NC, Li D, Bandyopadhyay S, Wang E, Merrill AH, Sewer MB. Acid ceramidase (ASAH1) represses steroidogenic factor 1-dependent gene transcription in H295R human adrenocortical cells by binding to the receptor. Mol Cell Biol. 2012;32(21):4419-31.

\section{Tables}

Table I The primer sequence

\begin{tabular}{ccc}
\hline Gene & Primer sequence & Number of bases \\
\hline StAR-F & 5'-CGTGGCTACTCAGCATCGA-3' & 19 \\
StAR-R & 5'-TGGGCACAGTTGGGAACA-3' & 18 \\
CYP19A1-F & 5'-TCACTGGCCTTTTTCTCTTGGT-3' & 22 \\
CYP19A1-R & 5'-GGGTCCAATTCCCATGCA- 3' & 18 \\
HSD3ß2-F & 5'-TCCACCCACCTGGCTTCAT-3' & 19 \\
HSD3ß2-R & 5'-GCAGGACCTGGGCTTGTG-3' & 18 \\
\hline
\end{tabular}

Table II Relationship between SF-1 expression and clinicopathological characteristics in 60 cases of endometrial carcinoma 


\begin{tabular}{|c|c|c|c|c|}
\hline Clinicopathological characteristics & $n$ & SF-1 expressio & & $\mathrm{P}$ \\
\hline Age & & & 0.67 & 0.423 \\
\hline$<$ 50year-old & 19 & $0.983 \pm 0.051$ & & \\
\hline$\geq$ 50year-old & 41 & $1.048 \pm 0.027$ & & \\
\hline Surgical pathological stages & & & 2.00 & 0.068 \\
\hline Stage I & 41 & $0.896 \pm 0.031$ & & \\
\hline Stage II $\square I V$ & 19 & $1.057 \pm 0.018$ & & \\
\hline Differentiation & & & 4.98 & 0.024 \\
\hline $\mathrm{G} 1$ & 34 & $0.705 \pm 0.041$ & & \\
\hline $\mathrm{G} 2$ & 14 & $0.963 \pm 0.039$ & & \\
\hline G3 & 12 & $1.197 \pm 0.035$ & & \\
\hline Pathological Type & & & 2.39 & 0.019 \\
\hline Endometroid adenocarcinoma & 42 & $0.797 \pm 0.036$ & & \\
\hline Special types $\mathrm{EC}^{\dagger}$ & 18 & 1. $134 \pm 0.029$ & & \\
\hline Depth of myometrial invasion & & & 0.84 & 0.395 \\
\hline$<1 / 2$ & 39 & $0.984 \pm 0.027$ & & \\
\hline$\geq 1 / 2$ & 21 & $1.058 \pm 0.031$ & & \\
\hline Lymph node metastasis & & & 1.29 & 0.312 \\
\hline yes & 7 & $1.013 \pm 0.017$ & & \\
\hline no & 53 & $0.924 \pm 0.022$ & & \\
\hline
\end{tabular}

†Special types of EC: serous carcinoma, mucinous carcinoma, clear cell carcinoma, carcinosarcoma, neuroendocrine carcinoma, mixed carcinoma, undifferentiated carcinoma

\section{Figures}

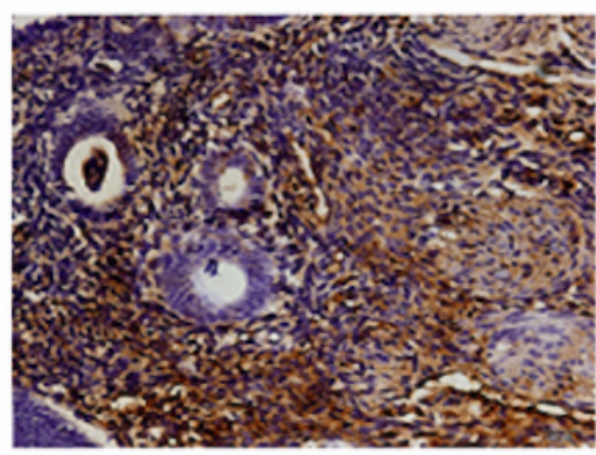

Endometrial cancer tissues

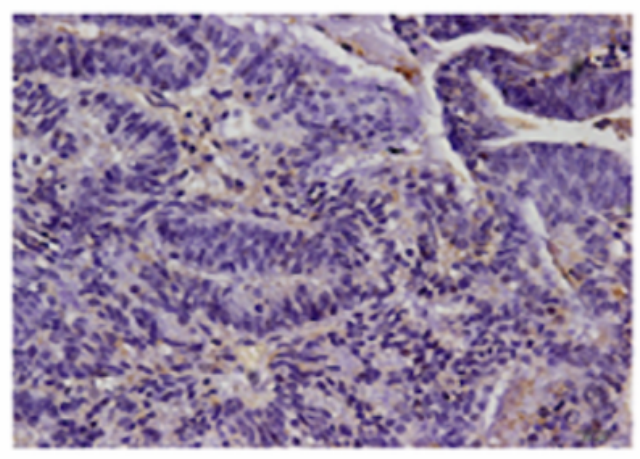

Paracancerous tissues

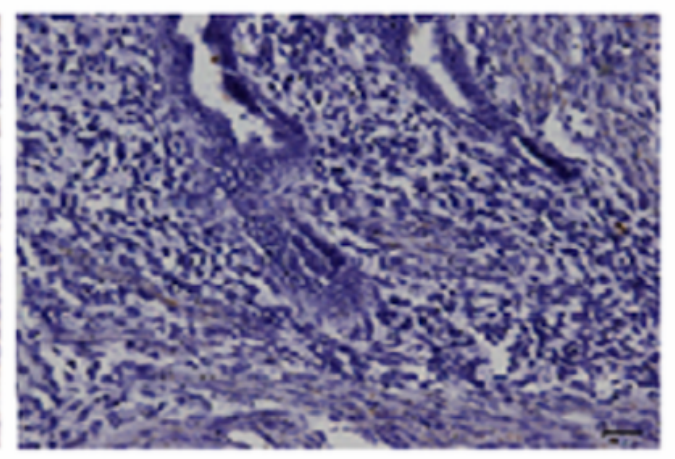

Normal endometrial tissues

\section{Figure 1}

expression of SF-1 protein detected by immunohistochemistry $\mathbb{I H C \times 2 0 0 \rrbracket}$ 


\section{$\begin{array}{llllllll}\text { T1 } & \text { T2 } & \text { T3 } & \text { T4 } & \text { T5 } & \text { T6 } & \text { T7 } & \text { T8 }\end{array}$}

\section{Endometrial cancer}

tissues

\section{Paracancerous} tissues

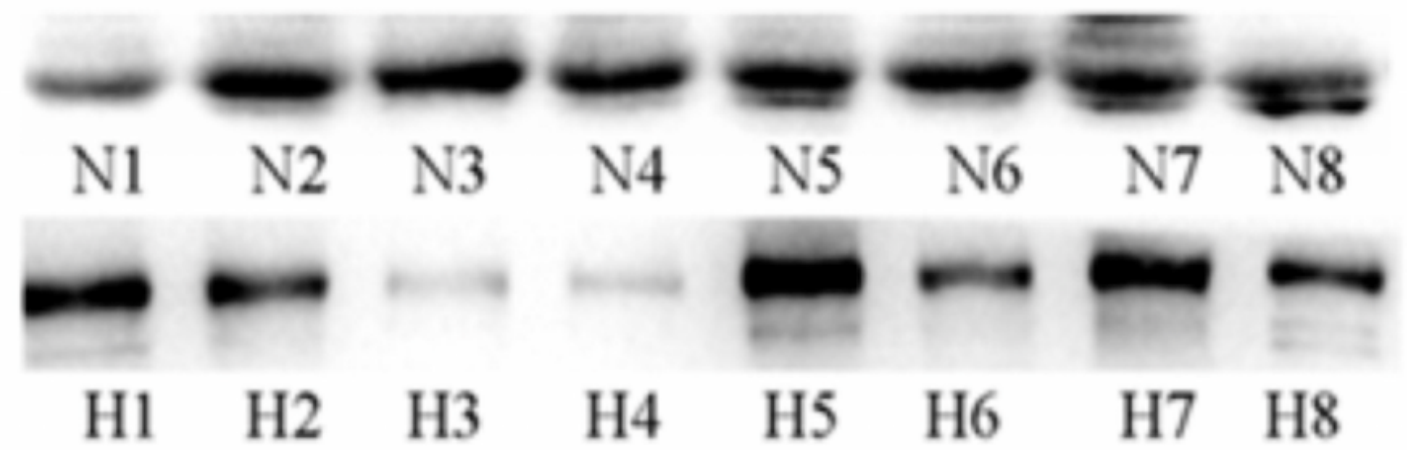

Normal endometrial tissues

\section{ACTB}

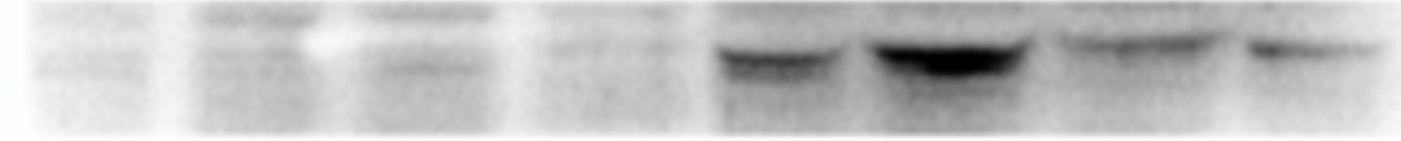

Figure 2

expression of SF-1 protein in different groups by Western Blot

A

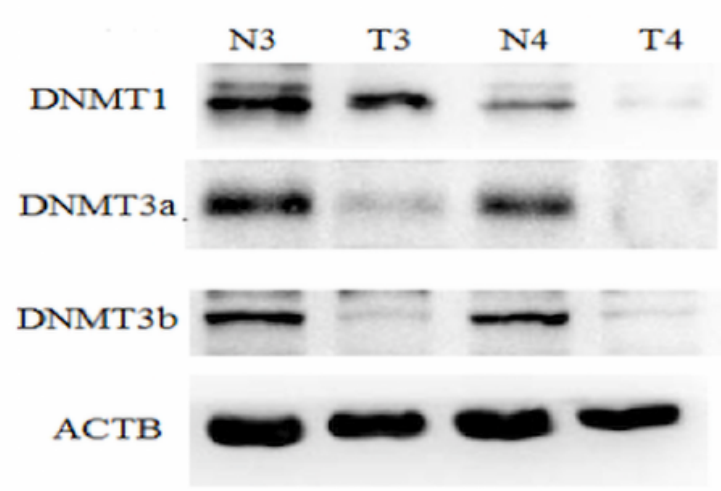

Figure3: expression of DNMTs in different groups by Western Blot

(A) The results of westem blot of DNMT1, DNMT3a and DNMT3b;

(B) Statistical results of the relative expression level of DNMTs in paracancerous

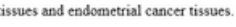

- the relative expression level of DNMTs in paracancerous tissues

$\square$ : the relative expression level of DNMTs in endometrial cancer tissues
B

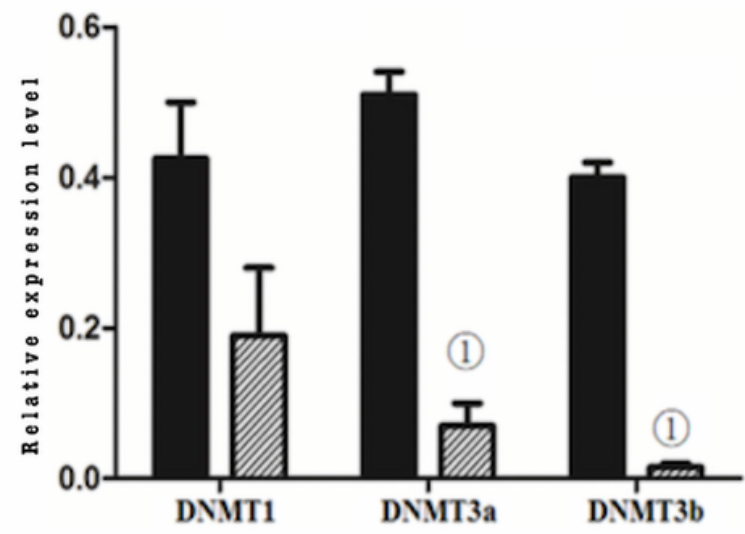

Paracancerous tissues

Endometrial cancer tissues

Figure 3

See image above for figure legend. 


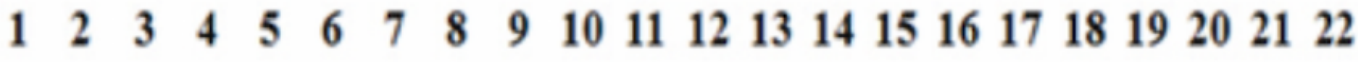
$000000000 \bullet 000000000000$

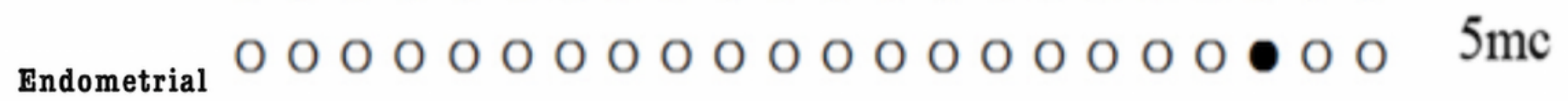 cancer

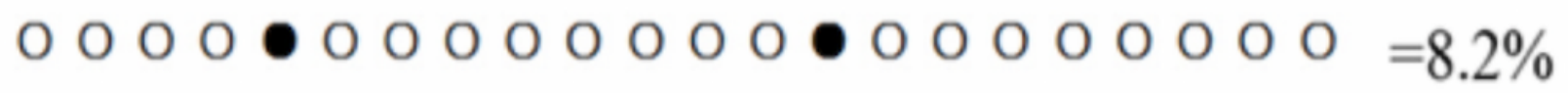
$00 \bullet 000 \bullet \bullet 0000 \bullet 000000000$
000000000000000000000

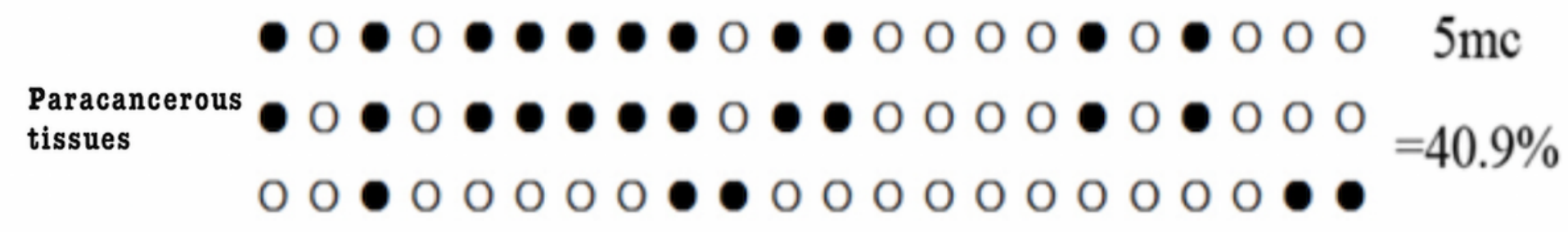

Figure 4

Methylation of SF-1 gene promotor in EC and paracancerous tissues $\bigcirc$ : methylated cytosine 0 : unmethylated cytosine mc: methylated cytosine 
A

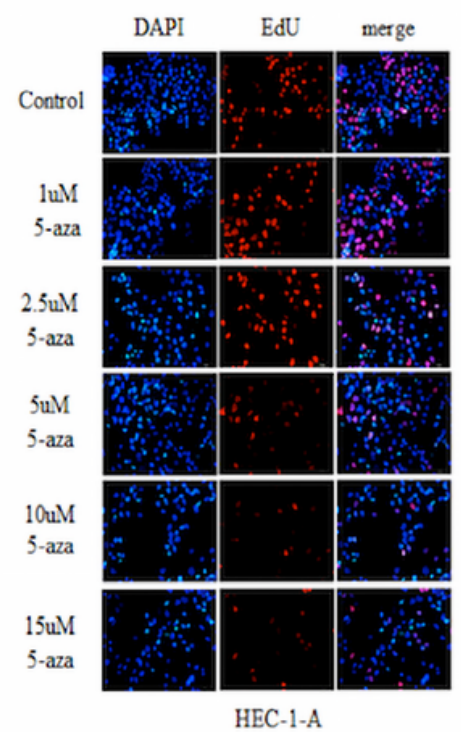

B

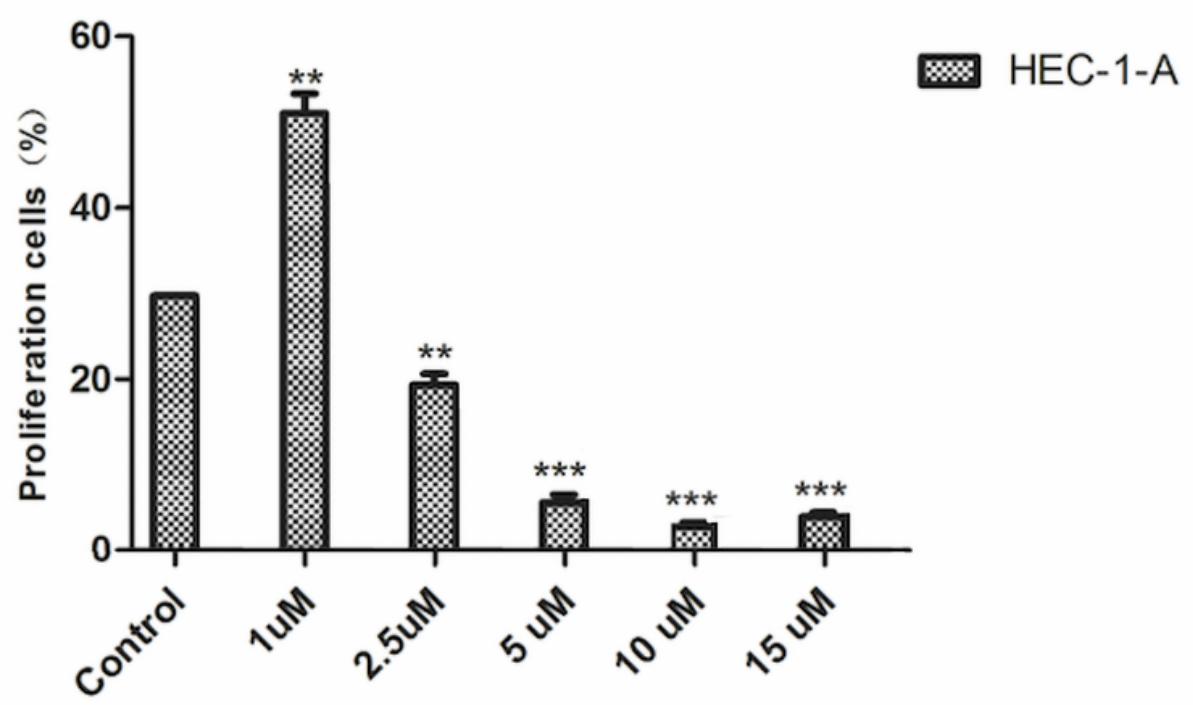

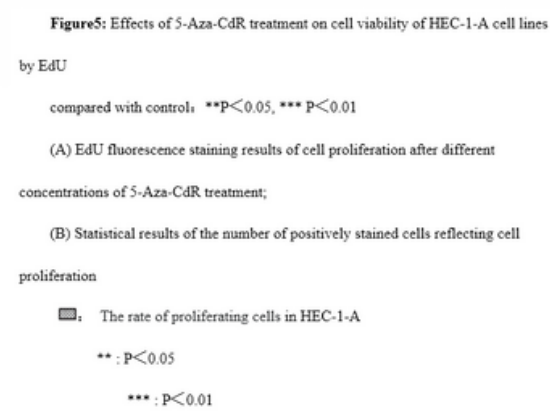

Figure 5

See image above for figure legend. 


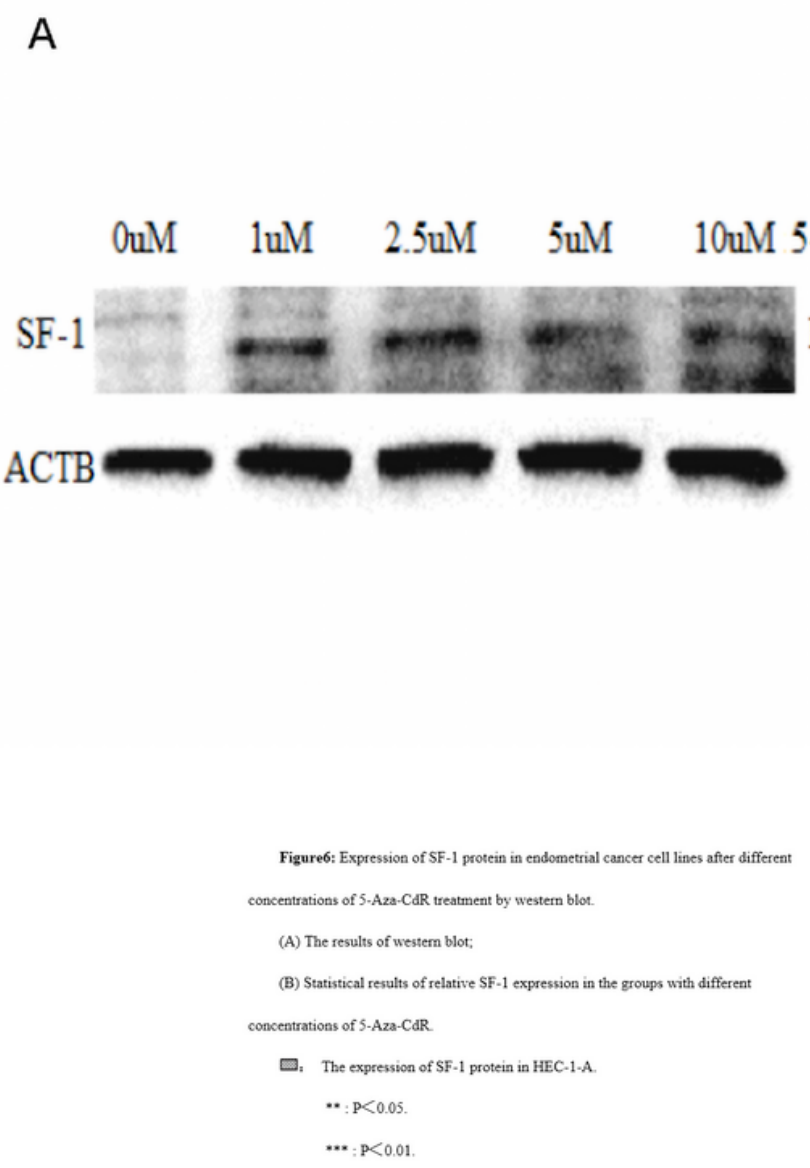

B

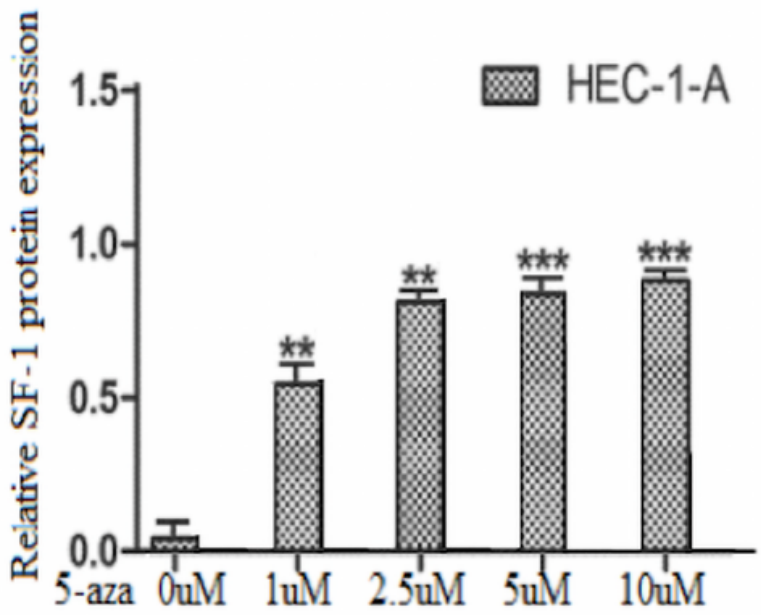

Figure 6

See image above for figure legend. 


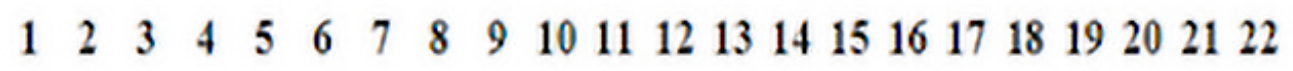

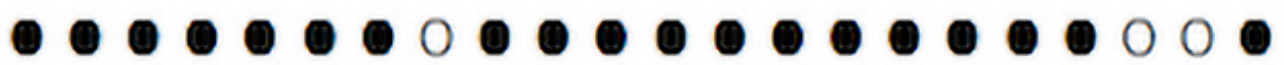

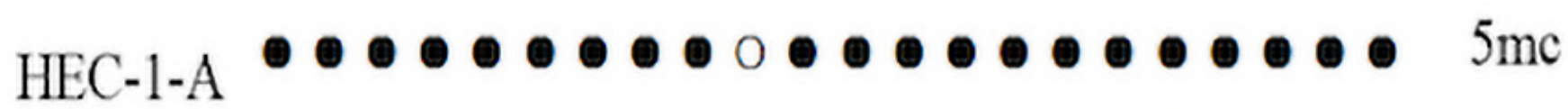
control

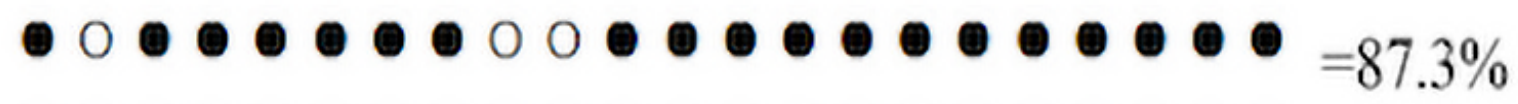

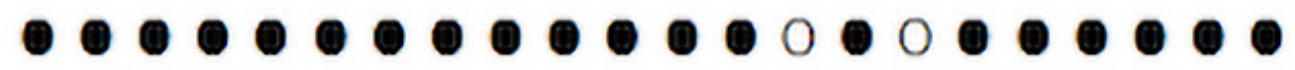
-

HEC-1-A

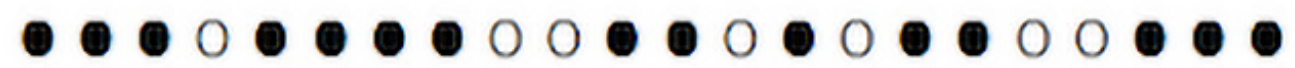

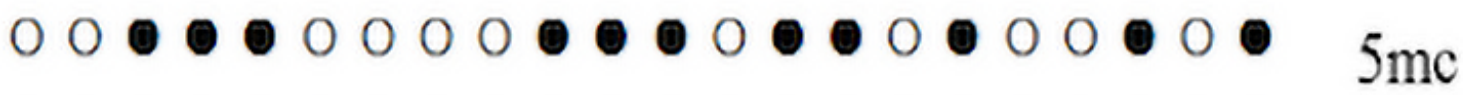
luM 5-aza $\bullet 0 \circ 0 \bullet 0 \bullet 0 \bullet \bullet \bullet \circ \bullet \circ \bullet \circ$

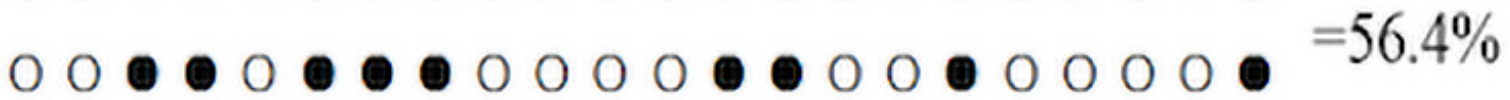
$\bullet \bullet 0 \bullet \bullet 0 \bullet \bullet 00 \bullet 00 \bullet \bullet 0 \bullet$

Figure 7

Methylation of SF-1 gene promotor in HEC-1-A cell line after 5-Aza-CdR by BSP. ๑: methylated cytosines. O: unmethylated cytosines. mc: methylated cytosine 

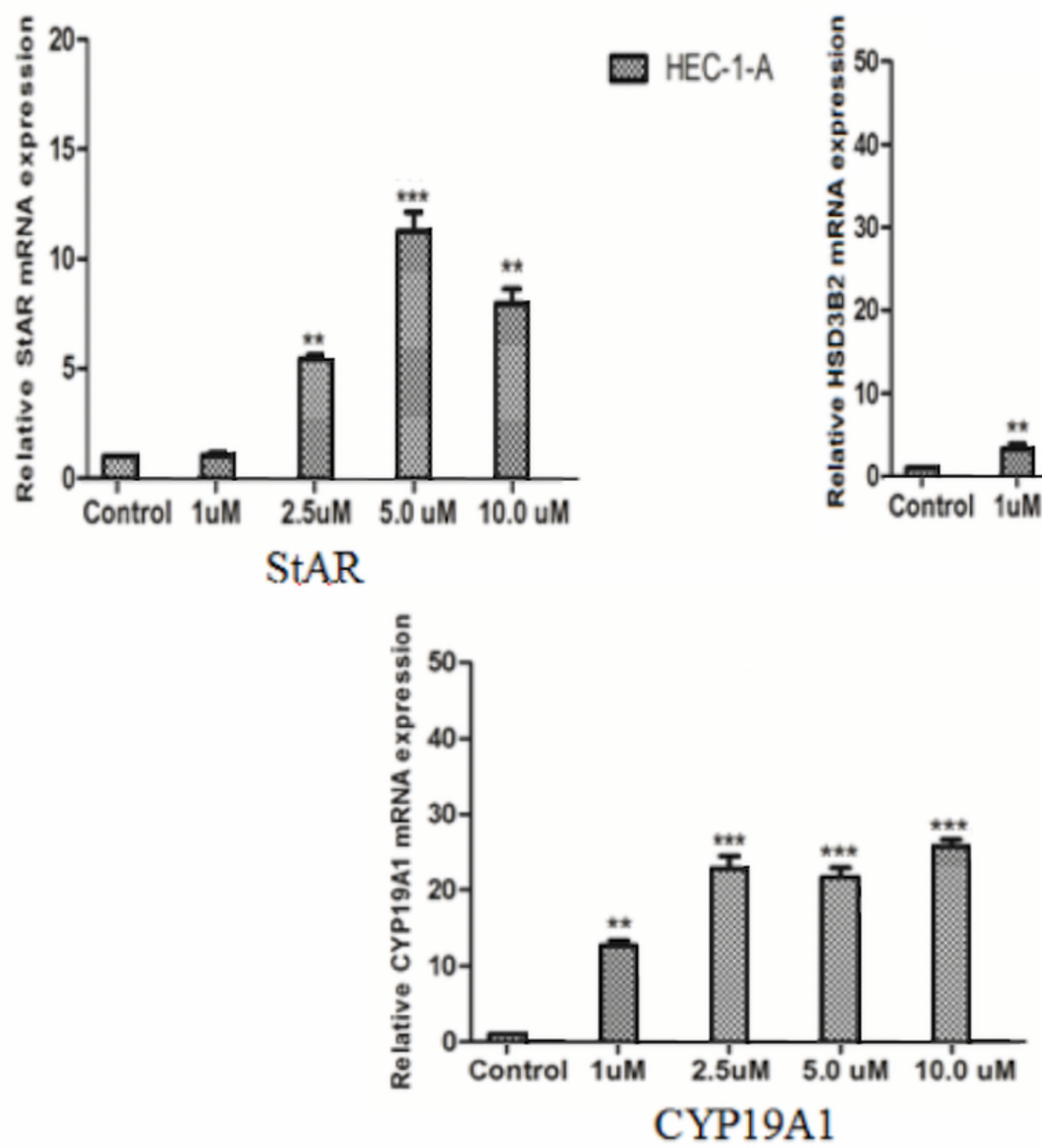

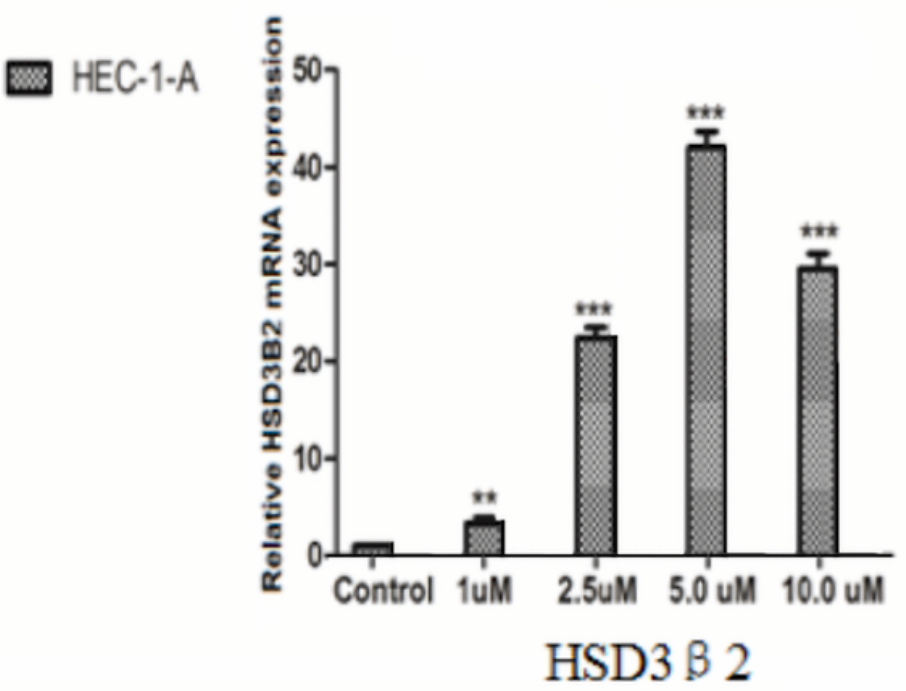

HEC-1-A 
A

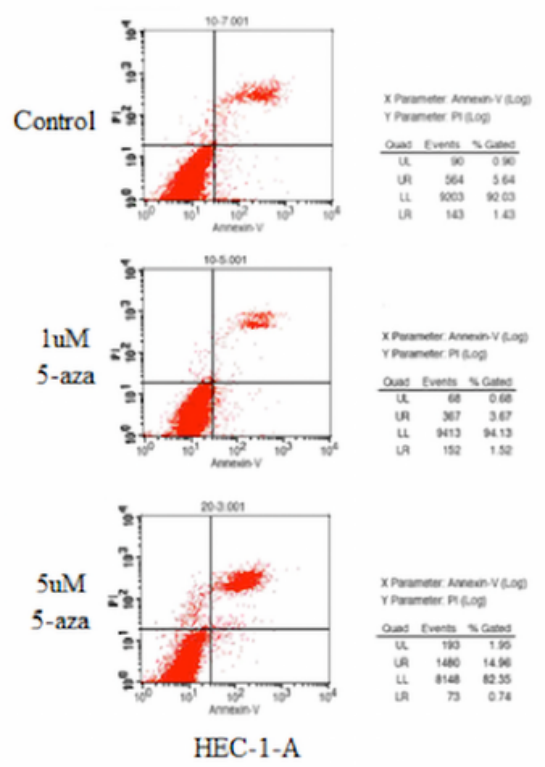

B

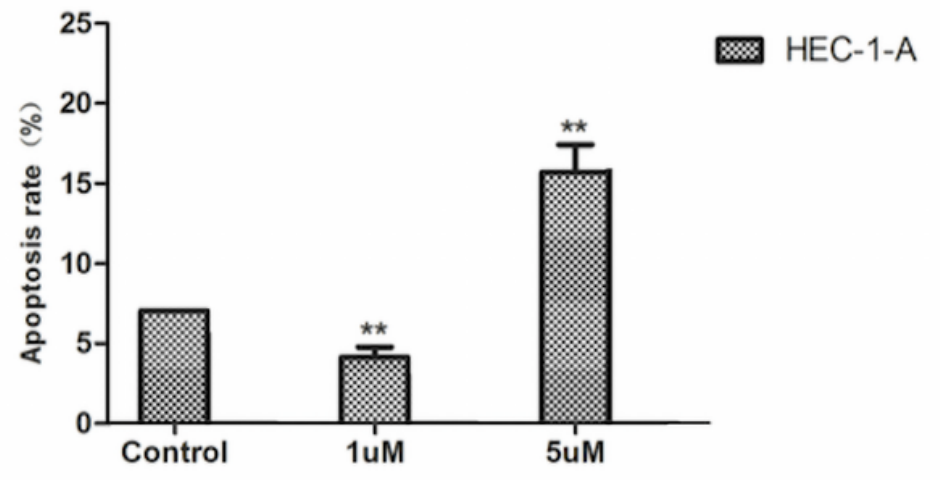

\section{Figure 9}

See image above for figure legend. 
A

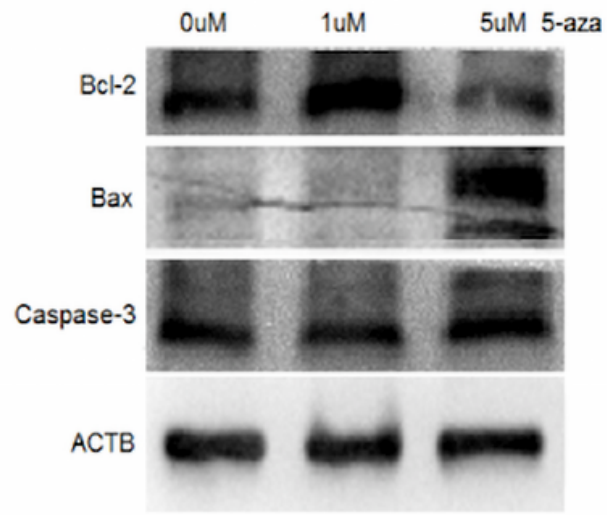

Figure 10: Expression of Bcl-2, Bax、Caspase-3 in HEC-1-A after different concentrations of 5 -Aza-CdR treatment.

(A) The results of western blot;

(B) Statistical results of expression of Bcl-2. Bax. Caspase-3 treated with

different concentrations of 5-Aza-CdR.

- The expression of Bcl-2 in HEC-1-A cell

Wa The expression of Bax in HEC.1-A cell.

$\square$. The expression of Caspase-3 in HEC-1-A cell

$*: \mathrm{p}<0.05$

$\cdots: \mathrm{p}<0.0$
B

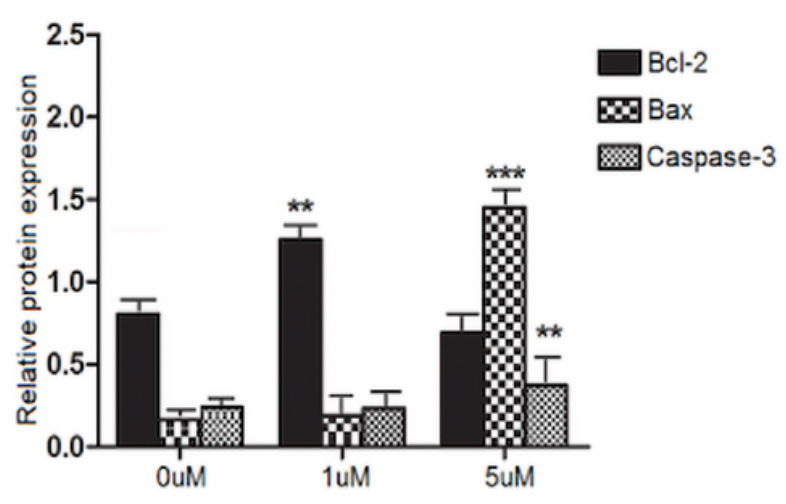

\section{Figure 10}

See image above for figure legend.

A

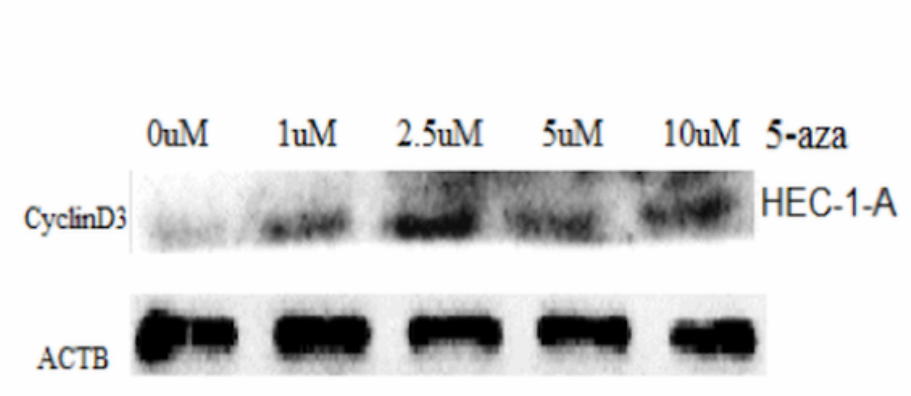

B

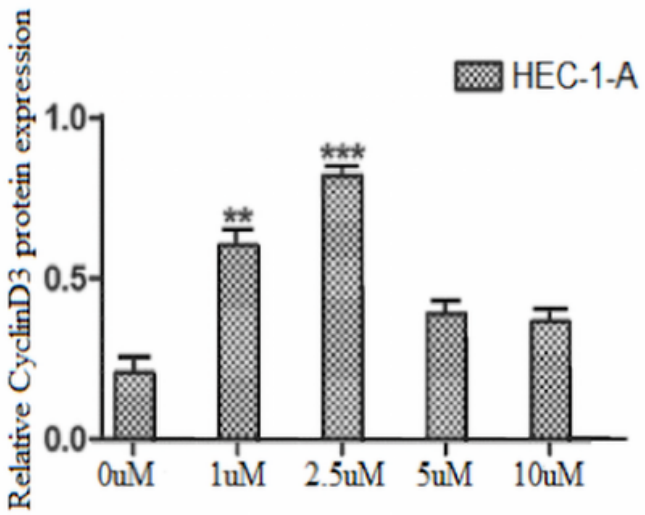

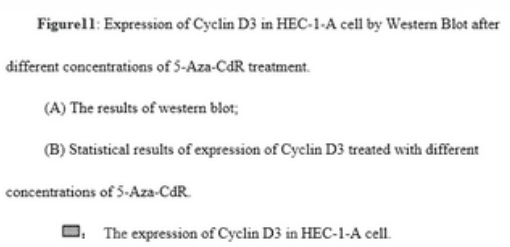




\section{Figure 11}

See image above for figure legend. 